\title{
Adaptive Underactuated Finger with Active Rolling Surface
}

\author{
Jesús M Gómez-de-Gabriel ${ }^{1}$, Member, IEEE, and Helge A Wurdemann ${ }^{2}$, Member, IEEE $^{2}$
}

\begin{abstract}
This paper presents the design, prototype and kinematic model of a new adaptive underactuated finger with an articulated skin/surface that is able to bend and, at the same time, provides active rolling motion along its central axis while keeping the finger configuration. The design is based on a planar chain of overlapping spherical phalanxes that are tendon-driven. The finger has an articulated surface made of an external chain of hollow universal joints that can rotate via its central axis on the surface of the internal structure. The outer surface provides a second active Degree of Freedom (DoF). The two actuators, driving the bending and/or rolling motion, can be used independently. A set of experiments have been included to validate and measure the performance of the prototype for the grasping and rolling actions. The proposed finger can be built with a different number of phalanxes and sizes. A number of these fingers can be arranged along a palm structure resulting in a multi-finger robotic grasper for applications that require adaptation and in-hand manipulation capabilities such as pHRI.
\end{abstract}

Index Terms-Robotic finger, underactuated manipulator, grasping, bending motion, rolling surface.

\section{INTRODUCTION}

W ITH the introduction of service, social and collaborative robots, autonomous systems will play an increasing role in our daily lives [1]. The healthcare sector provides a huge variety of applications for these robotic systems to provide social interaction [2], on the one hand, and assistance to support and help healthcare workers, patients and the elderly, on the other hand [3], [4]. In this sense, understanding physical Human-Robot Interaction (pHRI) in applications, such as rehabilitation [5], personal care [6], and exoskeletons [7], has become crucial to ensure safe interaction and promote trust. A continuously stable contact is one important factor that can contribute to a safe and trustworthy physical interaction between a human and robot [8]. In particular, challenges emerge in cases in which a robotic multi-finger end-effector needs to adjust or re-grasp, and it might be necessary to loosen the current grasp for a short time period to accommodate for this re-grasp [9].

Going beyond industrial end-effectors [10]-[13], researchers have proposed innovative end-effector designs that have the

Manuscript received: May 2, 2021.

This work has been partially funded by Universidad de Málaga and Ingeniería Uno S.L. under contract UMA-CEIATECH23, the Engineering and Physical Sciences Research Council (grant numbers: EP/S014039/1 and EP/V01062X/1) and the Royal Academy of Engineering (grant number: IAPP18-19\264).

${ }^{1}$ J.M. Gómez-de-Gabriel is with the Robotics and Mechatronics Group, University of Málaga, Spain. jesus.gomez@uma.es

${ }^{2}$ H.A. Wurdemann is with the Department of Mechanical Engineering, University College London, UK. h.wurdemann@ucl.ac.uk

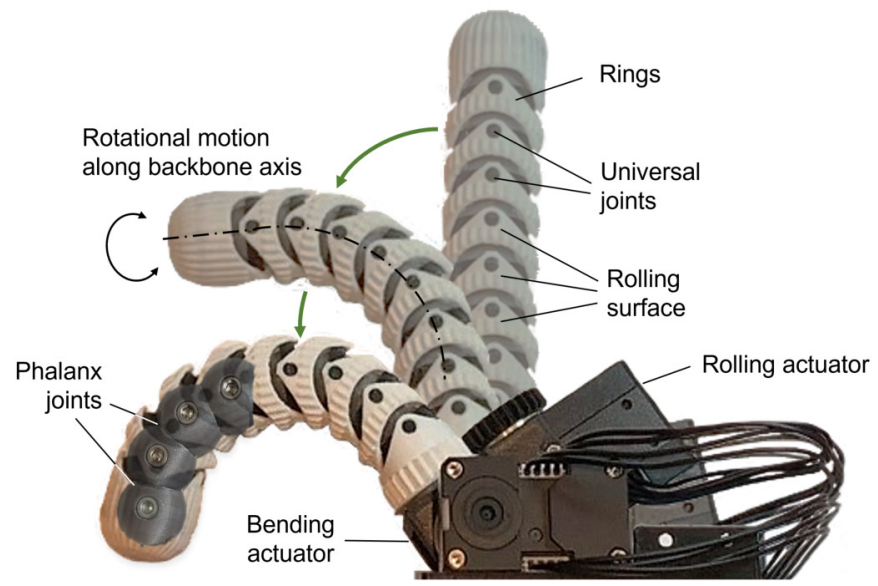

Fig. 1. Prototype of the adaptive underactuated finger with bending and rolling capabilities. While the finger flexes, its active skin is able to perform a rotational motion around its axis.

capability to maintain a stable grasp and, at the same time, manipulate objects in-hand. In [14], a gripper is made of multiple fingers with tension-controllable conveyor belts stretched along with each one. These active surfaces provide tangential forces that allow in-hand manipulation of objects with a variety of shapes by a rotational movement. A three-finger gripper with compact rollers at the fingertips is presented in [15], [16], where the end-effector provides rotational in-hand manipulation using two-Degree-of-Freedom spheres that provide three contact points with an object. In [17], [18], robotic fingers have been proposed with variable friction surfaces. Inspired by the soft tissue of a human finger pad allowing both gripping and sliding over objects during daily manipulations, gripping and sliding manipulation can be replicated by changing the friction properties along the fingers. A large re-orientations through the kinematics of the hand-object system alone, without the use of high fidelity contact sensors, complex control of active finger surfaces, or highly actuated fingers, has been achieved in [19]. These two-finger robotic grippers allow rotational inhand manipulation of objects.

Overall, end-effectors have been created with the capability to perform in-hand manipulation. Furthermore, some devices are able to move objects along the end-effector. These endeffectors clamp rigid objects with active surfaces. However, handling softer objects (e.g. human limbs) requires that each finger's active surface adapt bending around the object to provide a safe firm hold or grasp.

In this paper, we present an adaptive underactuated finger with an articulated skin/surface that is able to bend and, at 
the same time, provides active rolling motion while keeping the finger configuration. Our prototype is shown in Figure 1: One actuator drives the bending motion of the finger, whereas another actuator executes rotational motion along the finger's backbone axis. Multiple fingers can be arranged on a palm to create a robotic gripper that is able to move objects through the end-effector. Possible areas of application are personal care and assistive robotic systems for patients or the elderly. A robotic arm that is equipped with our end-effector would be able to grasp a limb to support the human to stand up from a sitting position. If the grasp position along the limb needs to be adjusted, our proposed device would be able to maintain the grasp while moving along the limb.

This paper is structured as follows: In Section II, the underlying concept of our adaptive underactuated finger is explained, introducing the articulated skin. Then, the mechanical design of the robotics finger is detailed. The mathematical formulation of the bending and rolling motion is presented in Section III. In Section IV, a number of experiments validate a multi-fingered end-effector made of three robotic fingers.

\section{PRototype DESIGN OF THE UNDERACTUATED FINGER AND INTERFACE INTO ROBOTIC END-EFFECTOR}

\section{A. The underlying concept of the rolling surface}

The innovative finger, shown in Figure 1, has been inspired by mechanisms such as the one in the hyper-redundant seriallinkage snake robot presented in [20], capable of tightly wrapping around and moving along a trunk using a rolling motion. Our device is able to execute bending and rolling motion: Bending motion is achieved by an internal series of universal joints, also known as Cardan joints. This underactuated chain of intersecting spheres with centres at the pivoting point of each joint is tendon-actuated by the bending actuator. The novelty of our finger lies in its rolling behaviour. A series of rings that are connected via universal joints is imposed on the internal bone structure. The rolling actuator transfers a rotational motion along the backbone axis of the finger.

\section{B. Proposed design of the finger}

A detailed exploded CAD illustration of the single finger is shown in Figure 2(a). The internal structure is made of nine intersecting phalanxes of spherical shapes. The spheres have a $12 \mathrm{~mm}$ diameter are connected to each other by universal joints resulting in a length of the internal finger structure of $126.5 \mathrm{~mm}$. A steel tendon with a diameter of $0.58 \mathrm{~mm}$ is then fed through embedded channels of each segment and connected to a capstan pulley system with a $10 \mathrm{~mm}$ mounted on a base. The phalanxes have been designed so that the joint bending values remain in the range $0 \leq q_{i} \leq q_{\max }=90^{\circ}$ to avoid any interference between the tendons and spherical joints. A servo motor (Dynamixel XM430-W210-T, ROBOTIS Inc., Korea) drives the pulley system clock- or anticlockwise, generating a planar bending motion, i.e., a finger flexion or extension, respectively.

The series of phalanxes are imposed with nine rings. Each ring has an external diameter of $25 \mathrm{~mm}$; hence, the overall length of the finger is $126.5 \mathrm{~mm}$. The rings are connected via universal joints. The base of this mechanism is driven via a gear system by another servo motor (Dynamixel XM430W210, ROBOTIS Inc., Korea). Again, this actuator will either allow the rings of the finger (the finger skin/surface) to either rotate clock- or anticlockwise.

Our prototype is made of PLA Thermoplastic using an FDM 3D printer (Mk2.3s from Prusa Research a.s., Praha, Czech Republic) and has a total weight (excluding servo motors) of $89 \mathrm{~g}$. The chosen design parameters are summarised in Table I. The minimum bending radius of the finger is about $21 \mathrm{~mm}$. The two Dynamixel servo motors are driven by a U2D2 control board achieving a maximum closing force of $19 \mathrm{~N}$ and rolling force of $14 \mathrm{~N}$. A PC running MATLAB (The Mathworks, Inc.) has been used to provide references to the actuators using the Dynamixel SDK and reading the desired variables.

\section{Integration of multiple fingers into a robotic end-effector}

Multiple fingers can be arranged and mounted on a palm. As shown in Figure 2(b), we have fabricated and assembled three fingers as described in Section II-B. Here, one finger is opposed by two fingers. When the bending actuators are activated, all fingers are flexing, resulting in a grasp of a ball, a foam tube or a human forearm for $\mathrm{pHRI}$, for instance. The rolling motion of each finger will result in a translational motion of prismatic- or cylindrical-like objects, e.g., when manipulating the foam tube or forearm.

\section{FORMULATION OF THE BENDING AND ROLLING MOTION}

The bending and rolling ability of the proposed finger can be actuated independently. These two active Degrees of Freedom (DoFs) are described by $q_{b}$ and $q_{r}$ and driven by two actuators with force and velocity control modes. This section presents both formulations for the kinematic modelling of the single underactuated finger.

\section{A. Tendon-driven backbone}

The internal structure (i.e., the nine intersecting phalanxes of spherical shapes) provides rigidity and support for the bending and closing motion of the finger. Two steel cable tendons are displaced by servo-controlled cable pulleys. The two opposing tendons join at the tip of the finger, providing control of the flexion and extension actions. Figure 3 illustrates details of the tendon routing within the internal phalanx structure. This routing does not interfere with the touching surfaces of the spherical links. The driving mechanism is inspired by the

TABLE I

ROLLING FINGER DESIGN PARAMETERS

\begin{tabular}{l|c|c} 
Parameter & Symbol & Value \\
\hline Number of phalanxes & $n$ & 9 \\
Phalanx diameter & $l$ & $12 \mathrm{~mm}$ \\
Base phalanx length & $l_{b}$ & $32.5 \mathrm{~mm}$ \\
External finger diameter & $d$ & $25 \mathrm{~mm}$ \\
Phalanx tendon radius & $r$ & $8 \mathrm{~mm}$ \\
Tendon pulley radius & $r_{p}$ & $10 \mathrm{~mm}$ \\
Max. phalanx bending joint angle & $q_{\max }$ & $20 \mathrm{deg}$.
\end{tabular}




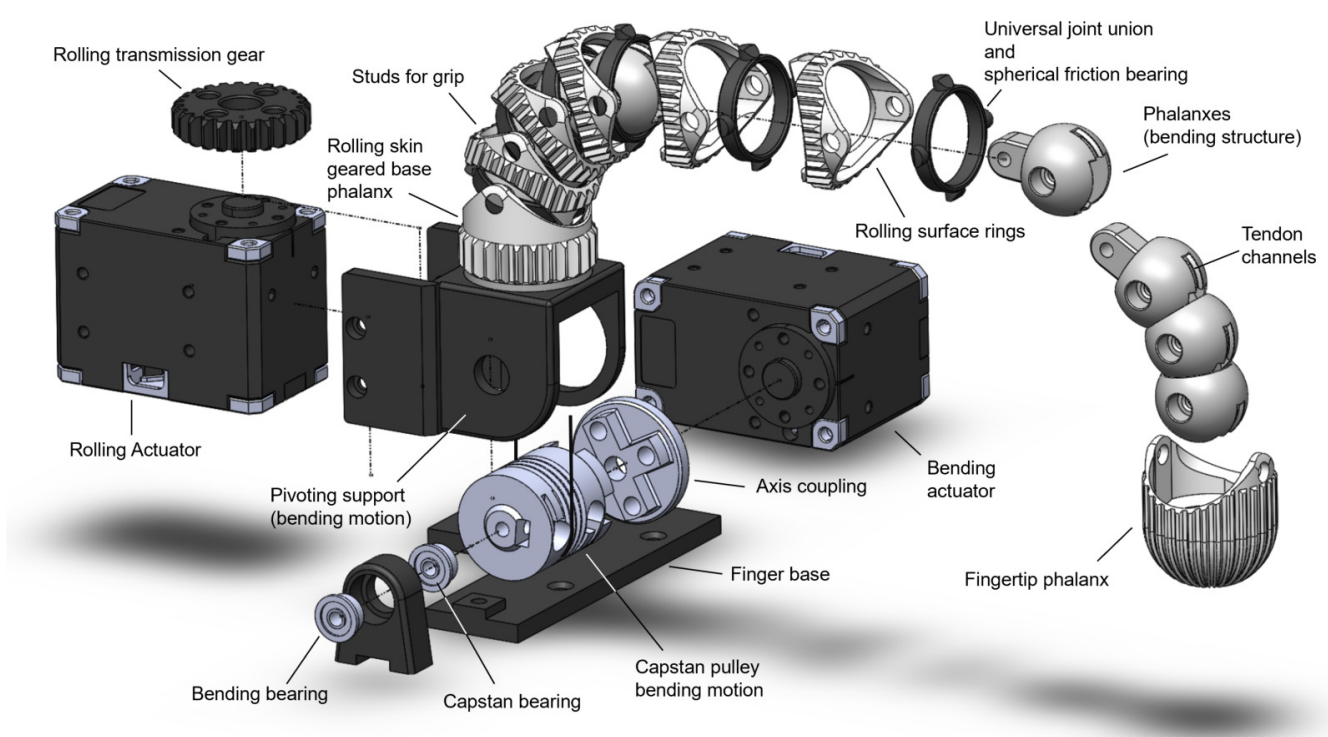

(a)
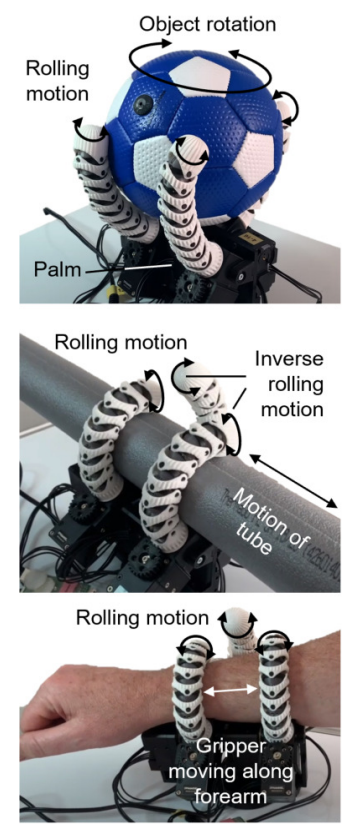

(b)

Fig. 2. (a) Exploded view of the adaptive underactuated finger with two degrees of freedom. The inner structure is made of a series of phalanxes that are connected via universal joints. Tendons are fed through the mechanism. A capstan pulley system will cause bending motion. The outer structure is made of a chain of rings connected via universal joints. This finger skin is able to rotate around the backbone axis of the finger. Two servo motors provide independent bending or rolling motion. (b) A gripper is made of three adaptive underactuated fingers mounted on a palm. The figures show how this type of end-effector can grasp objects. A rolling motion of each finger results in a translational motion of prismatic- or cylindrical-like objects.

Da Vinci mechanism [21]. Our design, however, does not use springs as the length of the two tendons in our finger design remains constant. Also, the first link shares its axis with the capstan pulley for an extended closing action. The open position of the backbone is straight and constrained by mechanical end-stops.

The tendon displacement depends on the actuator angle $q_{a}$, the finger joint values $q_{i}$ and the radius of the pulleys $r_{p}$ and $r_{t}$, according to (1).

$$
r_{p}\left(q_{a}-q_{1}\right)=r \sum_{i=2}^{n} q_{i}
$$

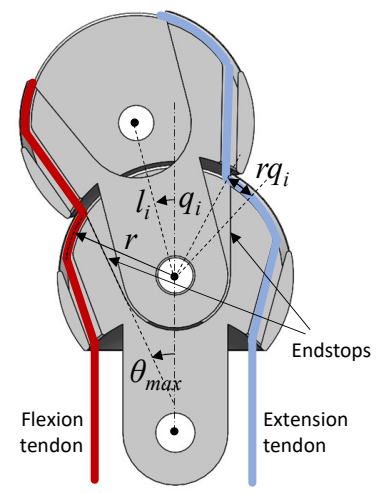

Fig. 3. Cross-sectional view of the Details of two spherical phalanxes providing details on the tendon routing. The flexion tendon is in red whereas the extension tendon is in blue colour. The rotational movement is limited by mechanical endstops to avoid any interference with the touching surfaces of the phalanxes. where $0 \leq q_{1} \leq 90^{\circ}$. The remaining joints are limited in their motion to avoid mechanical interference between any adjacent rings as $0 \leq q_{i} \leq q_{\max }, \forall i=2 . . n$ (see Table I).

1) Kinetostatic model: The kinetostatic model of the underactuated bending structure can be described by the Transfer and Jacobian matrices [21] as the equation of the virtual input and output yields in (2).

$$
t^{T} \omega_{a}=f^{T}(J \dot{Q})
$$

The velocities of the joint vector $Q$, can be computed from $\omega_{a}$ as well as the transfer matrix $T$ that describes the underactuated structure made of nine intersecting phalanxes of spherical shapes.

$$
t^{T} \omega_{a}=f^{T}\left(J T \omega_{a}\right)
$$

The transfer matrix $T \in \mathbb{R}^{n x n}$ in (3) can be written as shown in (4), considering the constraint in (1).

$$
T=\left|\begin{array}{ccccc}
1 & \frac{-r}{r_{p}} & \frac{-r}{r_{p}} & \ldots & \frac{-r}{r_{p}} \\
0 & 1 & 0 & \ldots & 0 \\
0 & 0 & 1 & \ldots & 0 \\
& & & \vdots & \\
0 & 0 & 0 & \ldots & 1
\end{array}\right|
$$

In (4), $t$ is the input torque vector from the bending actuator and $\omega_{a}$ the joint velocity vector as in (5).

$$
t=\left|\begin{array}{c}
\tau_{a} \\
0 \\
0 \\
\vdots \\
0
\end{array}\right| \quad \omega_{a}=\left|\begin{array}{c}
\dot{q}_{a} \\
\dot{q}_{2} \\
\dot{q}_{3} \\
\vdots \\
\dot{q}_{n}
\end{array}\right|
$$


In (5), $\tau_{a}$ is the actuator torque. The Jacobian matrix $J$ depends on the contact points and joint values $Q$. The tangential friction provides the Cartesian interaction forces at every contact point. To convert Cartesian forces at the contacts points, the transpose of matrix $F$ is added. Both $f$ and $J$ have the general structure for any underactuated finger that can be found in [21]. A stable grasp is defined as a contact situation where the force vector $F$ has positive values only, i.e., the phalanxes in contact with an object have positive contact forces, whereas the remaining phalanxes have zero contact force.

2) Bending radius: The maximum angle between adjacent phalanxes is limited by the mechanical interference between adjacent rings. This limits also the minimum radius of the internal surface of the finger, which can be defined as a function of the maximum joint angle between phalanxes $q_{\max }$, the distance between joints $l_{i}$ and the diameter of the rolling rings $d$, as described in (6) according to Figure 4.

$$
r_{\text {ext }}=\frac{1}{2}\left(l \frac{1+\cos \left(q_{i}\right)}{\sin \left(q_{i}\right)}-d\right)
$$

For the parameters of the prototype summarised in Table I, the minimum radius $r_{\text {ext }}$ for this prototype is $21.52 \mathrm{~mm}$ with a limited maximum joint value of $20^{\circ}$. The minimum radius sets the minimum size for an object, requiring full contact with every phalanx of a single finger. However, a gripper with opposed fingers, as described in Section II-C and shown in Figure 2(b), can grasp and manipulate smaller objects with partial contact of each finger.

\section{B. Outer rolling surface}

A single universal joint provides the mechanism for motion transmission between intersecting axes (as shown in Figure 2). The offset between the input and output angle of each joint is $90^{\circ}$. The input and output rings are the external rolling surface of the finger, and the output ring is the same as the input ring for the next joint.

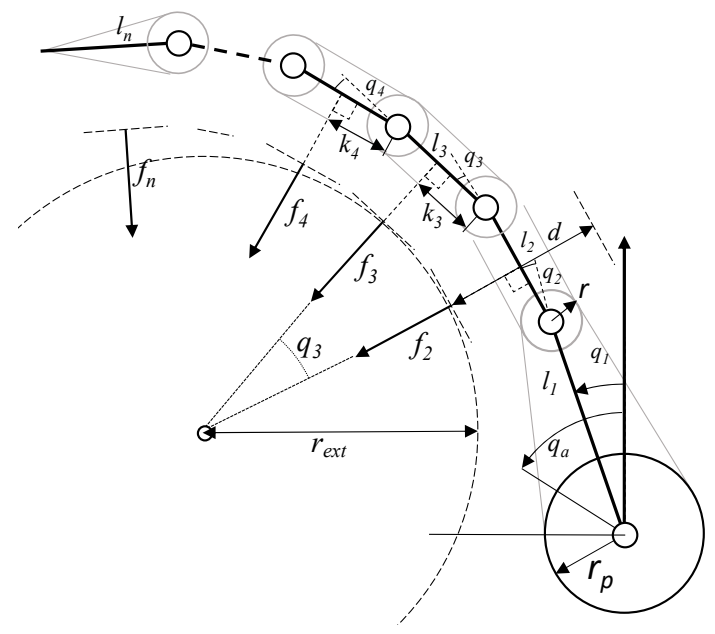

Fig. 4. Simplified kinetostatic model of the underactuated finger structure for the bending and closing action. The inner bending radius is set by the ring diameter, link length and joint angles.

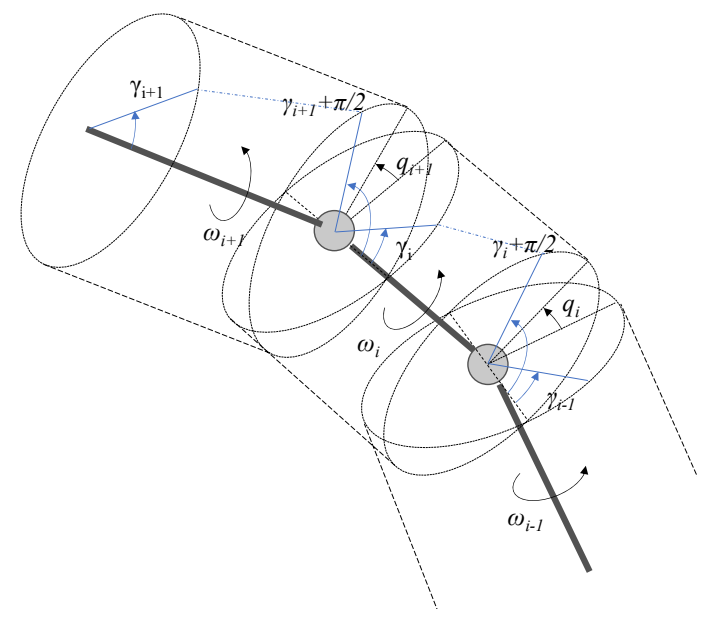

Fig. 5. Model of the velocity propagation in a double universal joint showing related links and angles. In non-CV joints, the rotational-velocity propagation depends on the angular position and bending angle.

1) Description of angular position: The angular position $q_{i}$ for each ring $\gamma_{i}$, rotating along the central axis $x_{i}$, can be expressed as in (7), shown in Figure 5.

$$
\tan \left(\gamma_{i}\right)=\frac{\tan \left(\gamma_{i-1}\right)}{\cos \left(q_{i}\right)}
$$

The angular movement transmission between adjacent rings depends on the cosine of the joint angle $q_{i}$. Thus, the surface speed is not constant. This has a limiting effect as the number $n$ of links (i.e., phalanxes) increases because the angle between joints is reduced. In our prototype, he have used $n=9$ links with joint limits $q_{\max }=20^{\circ}$. With a ring diameter of $25 \mathrm{~mm}$, the maximum relative displacement between adjacent ring surfaces is $\pm 1.78^{\circ}$ or $\pm 0.38 \mathrm{~mm}$. Finally, the maximum relative surface offset between the first and last ring is about $\pm 3.04 \mathrm{~mm}$.

2) Description of angular velocity: The velocity transmission between adjacent universal joints can be obtained by differentiating the expression in (7) and can be formulated as shown in (8)

$$
\omega_{i}=\frac{\omega_{i-1} \cos \left(q_{i}\right)}{1-\sin ^{2}\left(q_{i}\right) \cos ^{2}\left(\gamma_{i-1}\right)}
$$

where $\omega_{i+1}$ is the output velocity of a ring along its axis with respect to the velocity of the previous ring. Similarly, the rotation and surface velocities of two adjacent rings $\omega_{i+1}$ and $\omega_{i}$ have a sinusoidal relationship that depends on the joint angle $q_{i}$. The maximum difference in the surface velocity between the $1^{\text {st }}$ and $8^{\text {th }}$ ring is up to $54.6 \%$.

In a Constant Velocity transmission (CV), the angular velocity of the input shaft equals the velocity of the output (i.e., homokinetic transmission). However, the angular velocity propagation is not constant (non-CV) in a single universal (i.e., Cardan) joint [22] as shown in Figure 6.

3) Rolling Force: The relationship between the velocity at the base ring, which is directly driven by the actuator with a torque $\tau_{a r}$, and the subsequent rings of the finger can be described by (8). 


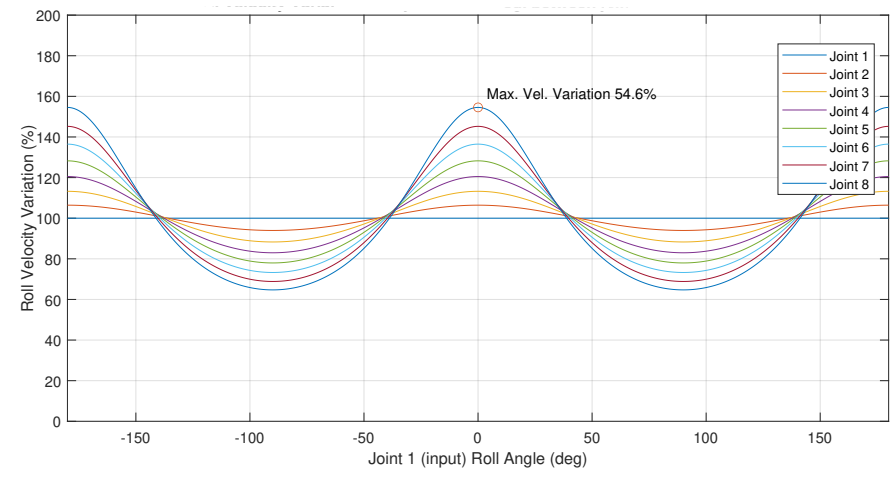

Fig. 6. Velocity variation in $\%$ between the base an subsequent rings assuming a constant angle $q_{i}=20^{\circ}$.

A Jacobian matrix $J$ is derived in (9) to relate torques of the configuration space $\tau_{a r}$ and the surface of the rings.

$$
T_{r}=J(Q, \Gamma) \tau_{a r}
$$

$T_{r}=\left[f_{r 1}, f_{r 2}, \ldots, f_{r n}\right]^{T}$ is the vector of torques for each ring, that depend on the values of $Q=\left[q_{1}, \ldots, q_{n}\right]$ and $\Gamma=\left[\gamma_{1}, \ldots \gamma_{n}\right]$. These values can be computed using (7) recursively. $\gamma_{1}$ can be obtained from the position sensor of the actuator. The components of the $n x 1$ Jacobian array $J=\left[j_{1}, J_{2}, \ldots J_{n}\right]$ can be computed recursively as in (8) shown in (10).

$$
\begin{aligned}
& J_{1}=1 \\
& J_{i}=\frac{\omega_{i-1} \cos \left(q_{i}\right)}{1-\sin ^{2}\left(q_{i}\right) \cos ^{2}\left(\gamma_{i-1}\right)}, \quad i=2, \ldots, n
\end{aligned}
$$

Finally, the tangential forces $F_{r}$ at the ring surfaces can be computed by multiplying the torques by the ring radius as in (11).

$$
F_{r}=\frac{d}{2} J(Q, \Gamma) \tau_{a r}
$$

The transmission of forces along the chain of universal joints is not homo-kinetic. Even when the actuator can provide uniform velocity at the base ring, a smooth movement or forces cannot be expected along the finger surface.

\section{EXPERIMENTAL PROTOCOL, SETUP, RESULTS AND DISCUSSION}

\section{A. Experimental protocol and setup}

To evaluate the performance of our adaptive underactuated finger, three experiments have been designed: One for the bending force and two for the rolling effect (force and speed).

Experiment 1 - Grasping force: As the bending backbone is an underactuated mechanism constrained by (1), the Cartesian forces depend on the actual joint angles, which rely on the shape of the grasped object. Without adding sensors, the individual values of the joints remain unknown. This experiment has been included to provide practical information on the expected Cartesian forces when grasping a cylindrical object (e.g., rod, bar, human arm). The dynamic measurement of the grasping force has been analysed according to the National Institute of Standards and Technology (NIST, U.S. Department of Commerce) performance metrics for robotic hands [23]. As shown in Figure 7(a), a force sensing split cylinder has been created using two load cells rated for $5 \mathrm{~kg}$, two HX711 instrumentation amplifiers, as well as integrated data acquisition circuits with digital outputs. A microcontroller (Arduino UNO) is used to periodically reading the data from the two data acquisition boards, converting raw readings into force units, and sending ASCII data to the host PC every $20 \mathrm{~ms}$. The cylinder housing was $3 \mathrm{D}$ printed with external diameters of $50 \mathrm{~mm}$ and two parts of an extension to increase its diameter up to $80 \mathrm{~mm}$ have also been built.

When measuring the grasping forces, the finger is located on a horizontal flat surface. A support structure for the force sensor has been designed that aligns the sensor's axis with the flat surface in either an orthogonal or parallel configuration, as shown in Figure 7(b). The actuator for the bending motion runs in an open-loop PWM mode, where the reference converts directly to the duty cycle signal to the embedded H-bridge. As the DC motor is mostly in stall state, the ratio PWM-torque has been considered linear according to the manufacturer's information: With a $12.0 \mathrm{~V}$ power supply, the servo provides a maximum torque of $3.0 \mathrm{Nm}$ at a PWM value of 885 with a current of $2.3 \mathrm{~A}$.

Each grasp is a sequence of torque ramps from 0 to $1 \mathrm{Nm}$. A total of four types of configurations of the force sensor have been considered: $50 \mathrm{~mm}$ and $80 \mathrm{~mm}$ diameter, as well as vertical versus horizontal force measurements, so that the grasping performance of the finger with two object sizes in two directions is understood.
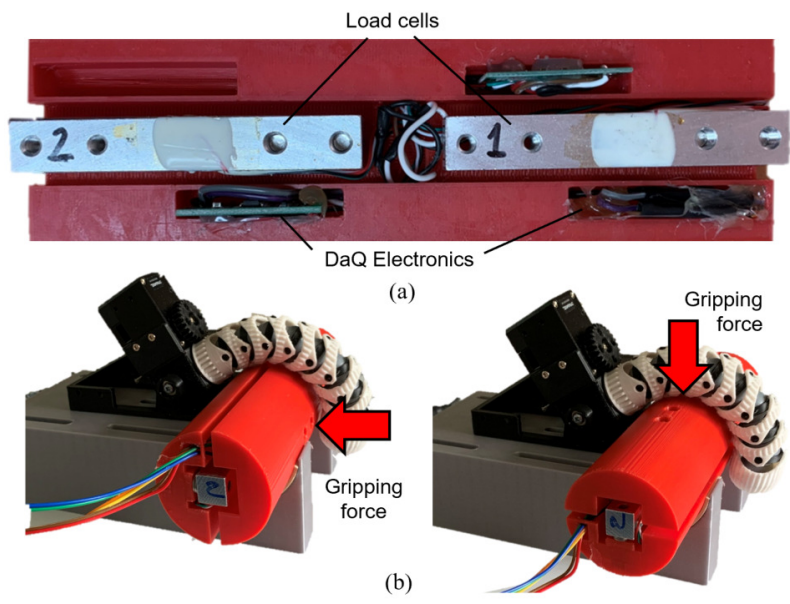

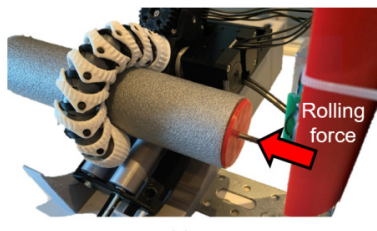

(c)

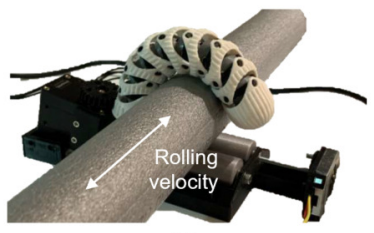

(d)
Fig. 7. (a) In Experiment 1, sensorised, cylindrical tubes with a $50 \mathrm{~mm}$ and $80 \mathrm{~mm}$ diameter have been created to analyse (b) vertical and horizontal grasping forces of our underactuated finger. (c) Experiment 2 analyses the pushing force introduced by the tangential forces of the rolling surface of the finger when cylindrical objects are grasped. (d) Experiment 3 measures the velocity at which cylindrical objects are moved by the rolling surface. 
Experiment 2 - Rolling force: This experiment has been designed to measure the tangential force that the active surface of the finger can apply to an object. This force depends on the friction at the contact surface between the finger and the object, which again is related to the grasping force. Hence, the rolling behaviour considering three different grasping torques have been studied. As shown in Figure 7(c), the bending actuator grasps a cylindrical object while the other actuator drives the rolling surface resulting in the object exerting a force onto a load cell. For this experiment, both actuators are controlled in open-loop PWM mode.

Two soft objects have been selected: insulation tubes made of polyethylene foam with a $40 \mathrm{~mm}$ and $60 \mathrm{~mm}$ external diameter. A force sensor has been implemented using a load cell with a similar data acquisition system to the one used in Experiment 1. As the tangential force depends on the friction between the finger rings of the rolling surface and the object, which in turn depends on the grasping force, three different grasping forces $(0.33 \mathrm{~N}, 0.67 \mathrm{~N}$ and $1.01 \mathrm{~N})$ have been used to measure the pushing force versus the rolling actuator torque for the two types of cylinders.

Experiment 3 - Rolling velocity: Experiment 3 has been designed to analyse the linear velocity of an object that is in contact or grasped by a single finger. The experimental setup is shown in Figure 7(d). Open-loop PWM control with a torque of $0.33 \mathrm{Nm}$ is applied for the bending behaviour of the finger, whereas closed-loop velocity control is implemented for the rolling surface.

To measure the rolling performance of a single finger, instead of using opposed fingers, two passive rollers opposite to the finger have been added. The rollers include an encoder to measure the sliding speed of the grasped object. A microcontroller read the output of an incremental encoder (AMT 102V, CUI Devices, USA) whereas provided velocity estimation every $20 \mathrm{~ms}$ and synchronisation to the host PC running a MATLAB script.

Six configurations have been considered: Two velocity references of $30 \mathrm{~mm} / \mathrm{s}$ and $60 \mathrm{~mm} / \mathrm{s}$ are tested grasping $40 \mathrm{~mm}$ and $60 \mathrm{~mm}$ polyethylene foam tubes. In addition, a pinch grasp, where the finger is mostly straight, has been evaluated.

\section{B. Experimental results}

Results for Experiment 1: Experiment 1 has been carried out nine times for the $50 \mathrm{~mm}$ and $80 \mathrm{~mm}$ diameter cylinder, respectively. The results, actuator torques versus closing forces, are shown in Figure 8. Figure 8(a) shows the grasping forces measured vertically for the cylinder with a $50 \mathrm{~mm}$ (blue) and a $80 \mathrm{~mm}$ diameter (red). Figure 8(b) displays the horizontal forces (attraction forces) for the cylinder with a $50 \mathrm{~mm}$ (blue) and a $80 \mathrm{~mm}$ diameter (red).

Despite all curves including noise, they can be approximate by a linear function. It is also worth noting that the measurement curves of the horizontal forces in Figure 8(b) remain zero up to $0.2 \mathrm{Nm}$ and $0.3 \mathrm{Nm}$, before the closing forces increase linearly. Furthermore, it can be concluded that the standard deviation (shaded area) among all measurements increase with a larger actuator torque being applied. When

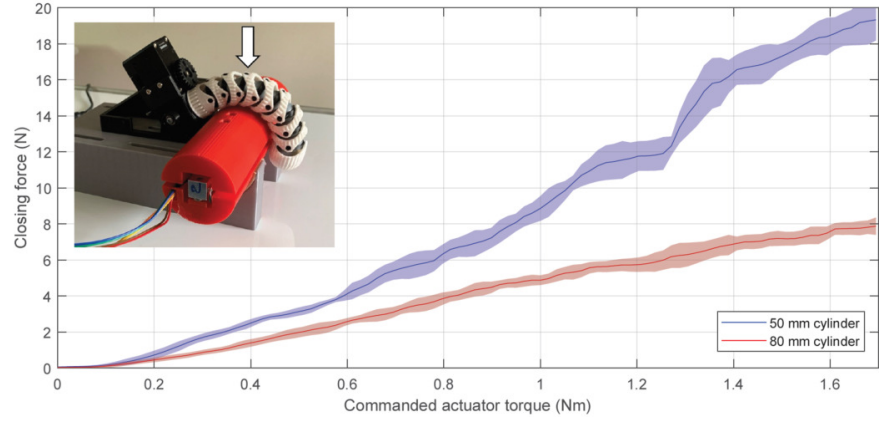

(a)

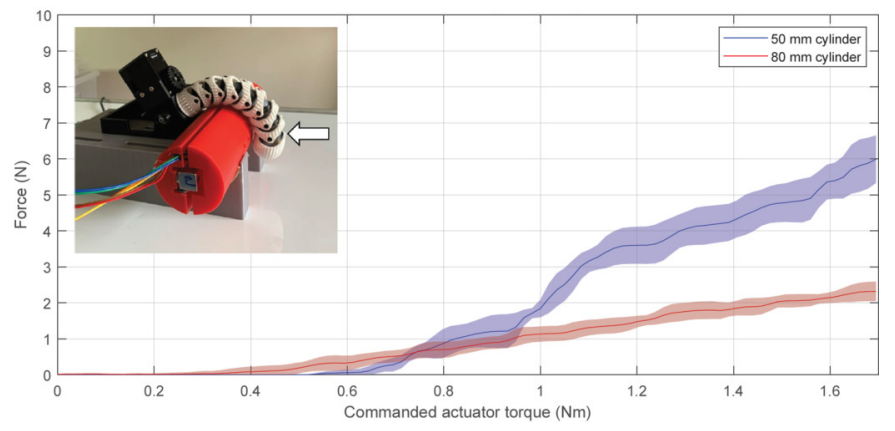

(b)

Fig. 8. Results of Experiment 1 (a) showing the grasping forces measured vertically and (b) showing the horizontal forces for the cylinder with a $50 \mathrm{~mm}$ (blue) and a $80 \mathrm{~mm}$ diameter (red).

comparing Figures 8(a) and (b), it is evident that the vertical forces are overall higher than the horizontal forces, when grasping the $50 \mathrm{~mm}$ and $80 \mathrm{~mm}$ diameter cylinder, respectively. At a $1 \mathrm{Nm}$ actuator torque, an maximum of $19 \mathrm{~N}$ and $7.8 \mathrm{~N}$ vertical force is achieved for the $50 \mathrm{~mm}$ and $80 \mathrm{~mm}$ diameter cylinder. The horizontal forces are $5.8 \mathrm{~N}$ and $2.3 \mathrm{~N}$. It can be seen that the closing forces measured grasping the $50 \mathrm{~mm}$ diameter cylinder are about twice as large as the forces using the $80 \mathrm{~mm}$ diameter cylinder.

Results for Experiment 2: The results of Experiment 2 with a $40 \mathrm{~mm}$ and $60 \mathrm{~mm}$ diameter cylinder are shown in Figure 9(a) and (b), respectively. In each case, three different grasping torques, i.e., $0.33 \mathrm{Nm}, 0.67 \mathrm{Nm}$, and $1.01 \mathrm{Nm}$ have been applied to the robotic finger. Over a period of $20 \mathrm{~s}$, the rolling actuator torque is increased linearly to $1.35 \mathrm{Nm}$. At the same time, the forces that the foam cylinder exerted to the load cell are recorded.

The measured forces applied to the load cell show an increasing periodic oscillation. The slope, at which the forces incline, is larger for larger grasping torques, reaching about $8 \mathrm{~N}$ and $13 \mathrm{~N}$ applying a grasping torque of $0.33 \mathrm{Nm}$ and $0.67 \mathrm{Nm}$ for a $40 \mathrm{~mm}$ diameter cylinder. For a $60 \mathrm{~mm}$ diameter cylinder, the maximum forces are above $6 \mathrm{~N}$ and $10 \mathrm{~N}$ for a grasping torque of $0.33 \mathrm{Nm}$ and $0.67 \mathrm{Nm}$.

For a grasping torque of $1.01 \mathrm{Nm}$ applied to a $40 \mathrm{~mm}$ diameter cylinder, however, the measured force stops oscillating at about $17 \mathrm{~s}$ and $1.15 \mathrm{Nm}$ rolling actuator torque. The force slightly decreases to about $7.8 \mathrm{~N}$ at a rolling actuator torque of $1.35 \mathrm{Nm}$. In the case of a $60 \mathrm{~mm}$ diameter cylinder, the force continues oscillating for the entire time period. However, 


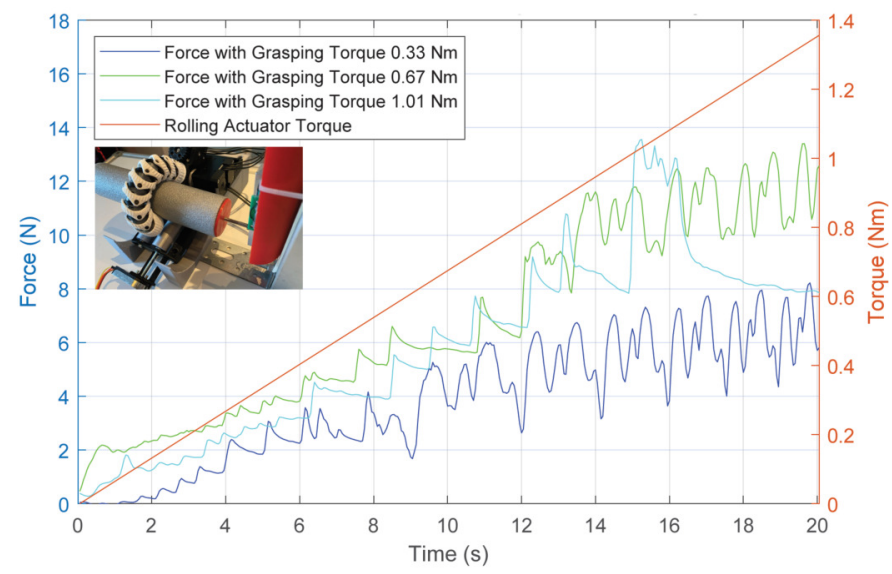

(a)

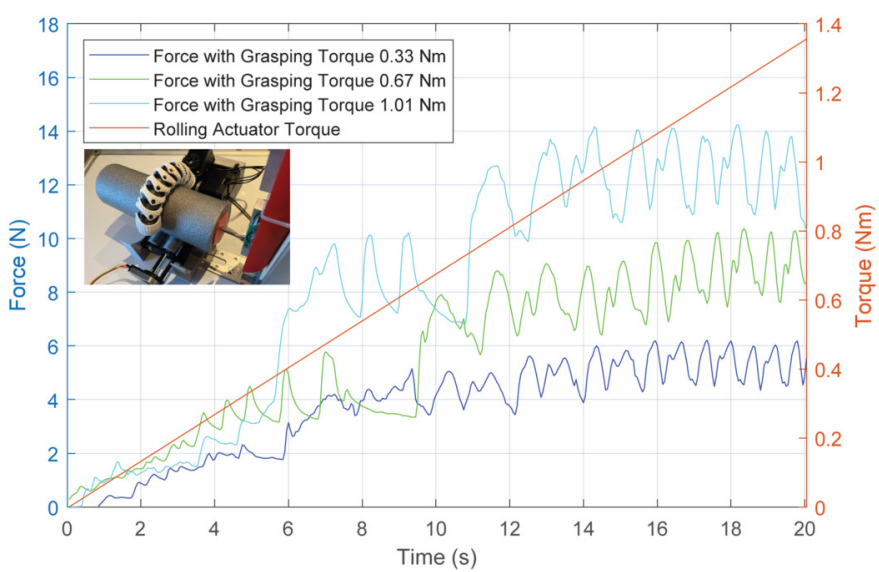

(b)

Fig. 9. Results of Experiment 2 measuring longitudinal forces applied by one finger to cylinders with a (a) $40 \mathrm{~mm}$ and (b) $60 \mathrm{~mm}$ diameter. Forces are monitored for grasping torques of $0.33 \mathrm{Nm}, 0.67 \mathrm{Nm}$, and $1.01 \mathrm{Nm}$, when the rolling actuator torque is increased linearly to $1.35 \mathrm{Nm}$.

after $12 \mathrm{~s}$ and a rolling torque of $0.9 \mathrm{Nm}$, the oscillating force stagnates at about $14 \mathrm{~N}$.

Results for Experiment 3: The results of Experiment 3 are presented in Figure 10. The yellow curves show the object velocities resulting from a tangential velocity of the rolling surface of $30 \mathrm{~mm} / \mathrm{s}$. In purple colour, the average speed is plotted. On the other hand, the blue curves display the velocities at a rotational speed of $60 \mathrm{~mm} / \mathrm{s}$. In Figure 10(a) and (b), polyethylene foam tubes with a diameter of $40 \mathrm{~mm}$ and $60 \mathrm{~mm}$ are used, respectively. Results for a straight finger configuration with the tip in touch with the tube is illustrated in Figure 10(c).

From the data, it is evident that all object velocities show a periodic oscillation and that the average object velocities are higher for larger rotational velocities of the rolling surface. Furthermore, the amplitudes of the oscillating velocities are larger for decreasing values of the bending diameter of the finger: For a rotational speed of $60 \mathrm{~mm} / \mathrm{s}$, the amplitude for a $40 \mathrm{~mm}$ diameter cylinder is approximately $0.15 \mathrm{~mm} / \mathrm{s}$, for a $40 \mathrm{~mm}$ diameter cylinder $0.10 \mathrm{~mm} / \mathrm{s}$ and about $0.03 \mathrm{~mm} / \mathrm{s}$ for a straight finger configuration, for instance.

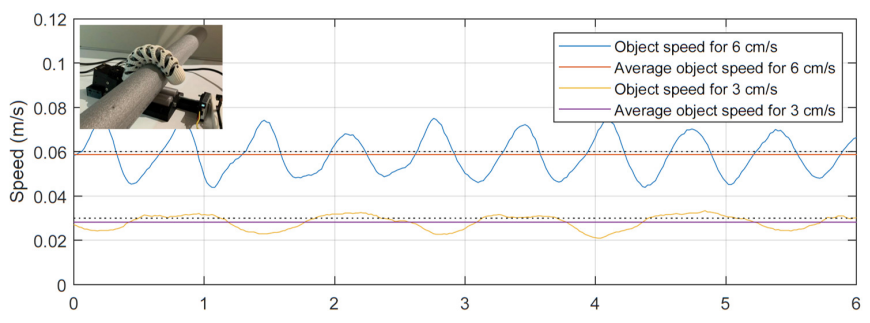

(a)

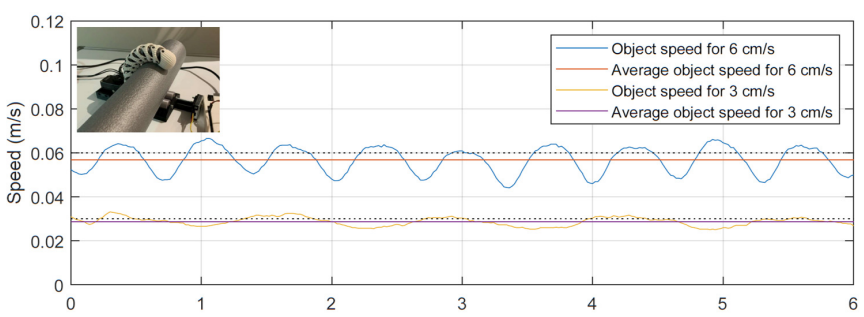

(b)

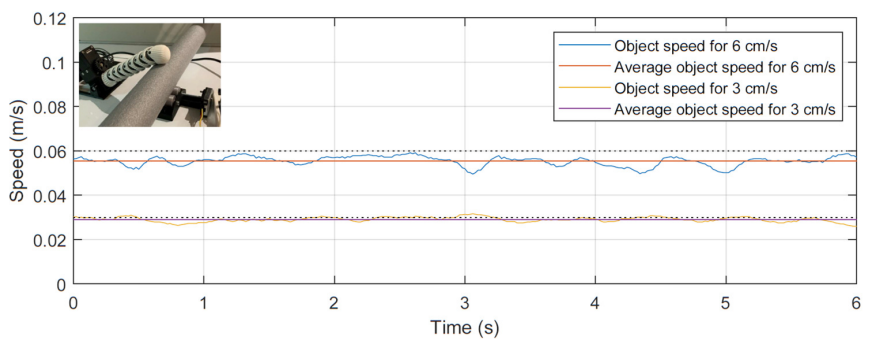

(c)

Fig. 10. Results of Experiment 3 analysing the linear velocity of an object that is in contact and grasped by a single finger. Two velocities of $30 \mathrm{~mm} / \mathrm{s}$ and $60 \mathrm{~mm} / \mathrm{s}$ are tested grasping a (a) $40 \mathrm{~mm}$ and (b) $60 \mathrm{~mm}$ diameter polyethylene foam tube. (c) A pinch grasp has been evaluated.

\section{Discussions}

In Experiment 1, grasping forces are applied to an object by the bending actuator, actuated by the tendon pulley system in the base (see Figure 2(a)), resulting in the phalanx joints bending. Forces are larger in horizontal direction for objects with larger diameters because the lever arm is shorter when grasping a cylinder with a $50 \mathrm{~mm}$ compared to a $80 \mathrm{~mm}$ diameter. This is true for the length of the lever arm between the base and the point where the vertical and horizontal forces are measured. Furthermore, increasing friction losses can be expected between the steel tendons and guiding channels when transmitting pulling forces to the distal phalanxes. A dead zone between zero and $0.2 \mathrm{~N} / 0.3 \mathrm{~N}$ for the horizontal-force experiments is found due to friction of the steel tendons until forces from the fingertip are exerted to the object.

In the Experiment-2 plots, the behavioural effects of the non-homokinetic Jacobian described in Section III-B3 can be seen in the form of periodic oscillations with an amplitude of about $0.2 \mathrm{~N}$. For the experiments with a $40 \mathrm{~mm}$ and $80 \mathrm{~mm}$ diameter cylinder and a grasping actuator torque of $1.01 \mathrm{Nm}$, the oscillation terminates at about $17 \mathrm{~s}$ and $1.15 \mathrm{Nm}$ rolling actuator torque, on the one hand, and stagnates in amplitude, on the other hand. When closing the finger at an actuator torque of $1.01 \mathrm{Nm}$, the compliant foam cylinder is squeezed, 
and, hence, the rolling skin fails to push the cylinder above a certain threshold. Also, it is worth noting that the $40 \mathrm{~mm}$ diameter cylinder is slightly below the minimum diameter described in Section III-A2 leading to mechanical interference with the rings. For the $80 \mathrm{~mm}$ diameter cylinder in Figure 9(b), there is no mechanical interference, but the pushing force stops growing above a torque of just $0.8 \mathrm{Nm}$.

The theoretical expression of the rolling velocity of the finger rings as explained in Section III-B2 is evident for constant rotational actuator velocities in the results of Experiment 3. Larger bending angles will result in larger amplitudes of the oscillation. In the last configuration, the finger remains mostly straight, so the amplitude of the oscillations in the velocity of the object is the lowest.

Despite the fact of having a non-CV transmission of the angular velocity of the rolling surface, the effects of the velocity variations are not visually perceived but can be measured as shown in Figure 10. Overall, the rolling effect of the device works as intended with good overall tangential speed and forces on the test objects.

\section{CONCLUSIONS}

This paper presented our work on the design, model and evaluation of a novel underactuated adaptive finger with an active surface that allows for axial rolling action when the finger is bending. While active surfaces in existing solutions concentrate on small contact areas, our proposed solution covers the full surface of the finger. With null rolling velocity, the finger behaves as a conventional adaptive finger.

Grippers with in-hand manipulation capabilities can be built with a combination of rolling fingers. When all the fingers roll in the same direction, grasped objects rotate along the gripper's z-axis. When opposed fingers have complementary rolling velocity, the objects move linearly. We envision applications of our proposed gripper in the area of pHRI, e.g., for assistive robotic systems for the elderly.

As the active surface has been designed as a series of hollow universal joints, the angular velocity propagation between rings is not constant. This has effects on the velocities and forces, which needs to be included in the mathematical model. Nevertheless, experimental results have been presented showing the real effects of this non-homokinetic behaviour in different conditions. A different arrangement of the Cardan joints can be used to build CV transmissions. However, this design would make the resulting design less compact and increasing the minimum bending radius.

The prototype has been designed and manufactured using low-cost 3D printing technologies, materials and components. With the use of advanced materials and manufacturing methods, structures with lower frictions can be made to improve the current prototype and obtain increased performance measurements.

\section{REFERENCES}

[1] A. Cherubini, R. Passama, A. Crosnier, A. Lasnier, and P. Fraisse, "Collaborative manufacturing with physical human-robot interaction," Robotics and Computer-Integrated Manufacturing, vol. 40, pp. 1-13, 2016.
[2] S. Rossi, F. Ferland, and A. Tapus, "User profiling and behavioral adaptation for hri: A survey," Pattern Recognition Letters, vol. 99, pp. 3$12,2017$.

[3] K. Krishnaswamy, S. Moorthy, and T. Oates, "Survey data analysis for repositioning, transferring, and personal care robots," in International Conference on Pervasive Technologies Related to Assistive Environments, pp. 45-51, 2017.

[4] R. Krishnan and S. Pugazhenthi, "Mobility assistive devices and selftransfer robotic systems for elderly, a review," Intelligent Service Robotics, vol. 7, p. 37-49, 2017.

[5] A. Stilli, A. Cremoni, M. Bianchi, A. Ridolfi, F. Gerii, F. Vannetti, H. Wurdemann, B. Allotta, and K. Althoefer, "Airexglove - a novel pneumatic exoskeleton glove for adaptive hand rehabilitation in poststroke patients," in IEEE International Conference on Soft Robotics, p. $579-584,2018$

[6] Y. Ansari, M. Manti, E. Falotico, Y. Mollard, M. Cianchetti, and C. Laschi, "Towards the development of a soft manipulator as an assistive robot for personal care of elderly people," International Journal of Advanced Robotic Systems, vol. 14, no. 2, pp. 1-17, 2017.

[7] Z. Li, B. Huang, Z. Ye, M. Deng, and C. Yang, "Physical humanrobot interaction of a robotic exoskeleton by admittance control," IEEE ransactions on Industrial Electronics, vol. 65, pp. 9614-9624, 2018.

[8] S. Bowyer and F. R. y Baena, "Dissipative control for physical human-robot interaction," IEEE Transactions on Robotics, vol. 31, p. 1281-1293, 2015.

[9] J. M. Gandarias, F. Pastor, A. J. Muñoz-Ramírez, A. J. García-Cerezo, and J. M. Gómez-de-Gabriel, "Underactuated gripper with forearm roll estimation for human limbs manipulation in rescue robotics," in IEEE/RSJ International Conference on Intelligent Robots and Systems, pp. 5937-5942, 2019.

[10] L. U. Odhner, L. P. Jentoft, M. R. Claffee, N. Corson, Y. Tenzer, R. R. Ma, M. Buehler, R. Kohout, R. D. Howe, and A. M. Dollar, "A compliant, underactuated hand for robust manipulation," The International Journal of Robotics Research, vol. 33, no. 5, pp. 736-752, 2014.

[11] L. Pelliccia, M. Schumann, M. Dudczig, M. Lamonaca, P. Klimant, and G. D. Gironimo, "Implementation of tactile sensors on a 3-fingers robotiq adaptive gripper and visualization in vr using arduino controller," Conference on Intelligent Computation in Manufacturing Engineering, vol. 67, pp. 250-255, 2018.

[12] "Rightpick2 data sheet." https://www.righthandrobotics.com/resources/ rightpick2-data-sheet. Accessed: 2021-05-01.

[13] L. Beddow, H. Wurdemann, and D. Kanoulas, "A caging inspired gripper using flexible fingers and a movable palm," in IEEE/RSJ International Conference on Intelligent Robots and Systems, 2021.

[14] N. Govindan and A. Thondiyath, "Design and analysis of a multimodal grasper having shape conformity and within-hand manipulation with adjustable contact forces," Journal of Mechanisms and Robotics, vol. 11, no. 5, 2019.

[15] S. Yuan, A. D. Epps, J. B. Nowak, and J. K. Salisbury, "Design of a roller-based dexterous hand for object grasping and within-hand manipulation," in IEEE International Conference on Robotics and Automation, pp. 8870-8876, 2020.

[16] S. Yuan, L. Shao, C. L. Yako, A. Gruebele, and J. K. Salisbury, "Design and control of roller grasper v2 for in-hand manipulation," 2020.

[17] H. X. Trinh, V. A. Ho, and K. Shibuya, "Theoretical foundation for design of friction-tunable soft finger with wrinkle's morphology," IEEE Robotics and Automation Letters, vol. 4, no. 4, pp. 4027-4034, 2019.

[18] A. J. Spiers, B. Calli, and A. M. Dollar, "Variable-friction finger surfaces to enable within-hand manipulation via gripping and sliding," IEEE Robotics and Automation Letters, vol. 3, no. 4, pp. 4116-4123, 2018.

[19] W. G. Bircher, A. M. Dollar, and N. Rojas, "A two-fingered robot gripper with large object reorientation range," in IEEE International Conference on Robotics and Automation, pp. 3453-3460, 2017.

[20] C. Wright, A. Buchan, B. Brown, J. Geist, M. Schwerin, D. Rollinson, M. Tesch, and H. Choset, "Design and architecture of the unified modular snake robot," in IEEE International Conference on Robotics and Automation, pp. 4347-4354, 2012.

[21] L. Birglen, T. Laliberté, and C. M. Gosselin, Underactuated robotic hands, vol. 40. Springer, 2007.

[22] D. Farhadi Machekposhti, N. Tolou, and J. L. Herder, "A Review on Compliant Joints and Rigid-Body Constant Velocity Universal Joints Toward the Design of Compliant Homokinetic Couplings," Journal of Mechanical Design, vol. 137, 03 2015. 032301.

[23] J. Falco, K. Van Wyk, and E. Messina, "Performance metrics and test methods for robotic hands," NIST, Tech. Rep. DRAFT NIST Special Publication, vol. 1227, 2018. 